\title{
Icosahedra Constructed from Congruent Triangles
}

\author{
E. N. Miller \\ Department of Mathematics, University of California, \\ Berkeley, CA 94720, USA \\ enmiller@math.berkeley.edu
}

\begin{abstract}
It is possible to construct a figure in three dimensions which is combinatorially equivalent to a regular icosahedron, and whose faces are all congruent but not equilateral. Such icosamonohedra can be convex or nonconvex, and can be deformed continuously. A scalene triangle can construct precisely zero, one, or two convex icosamonohedra, and each occurs. Demonstrated here are two explicit convex examples, the first of which is the unique such object constructed from scalene right triangles, proving a conjecture of Banchoff and Strauss.
\end{abstract}

\section{Introduction: Monohedra}

A monohedron is essentially a 3-polyhedron whose faces are all congruent. The following conventions allow for more precision in certain cases where adjacent faces are coplanar. A polyhedron is bounded and three-dimensional, but not necessarily convex (e.g., for Corollary 5.2). Each polyhedron $P$ in this paper is endowed with a polytopal subdivision [Z, Example 5.2] of each of its facets, although these are often trivial. The terms facet and ridge refer to maximal and one-dimensional cells of the unsubdivided boundary complex of $P$. These are to be distinguished from the faces and edges of $P$, which refer to maximal and one-dimensional cells of any of the facet subdivisions. In what follows, congruences are not required to preserve orientation.

Definition 1.1. With the conventions above, a polyhedron $P$ with its facet subdivisions is called a monohedron if all of its faces are congruent to a single face $T$. $P$ is said to have protoface $T$.

Monohedra, particularly convex monohedra, have been objects of interest since ancient times, when the five Platonic solids were first described. Three of these solids have triangular faces, and it would seem natural to attempt a classification of convex 
monohedra with equilateral triangular faces; yet this elementary task was published only in 1947 by Freudenthal and van der Waerden [FW]. The step taken here is to forgo the requirement that the faces be equilateral.

In particular, this paper deals with scalene icosamonohedra: monohedra combinatorially equivalent to the regular icosahedron with scalene triangular protoface. Combinatorial issues are dealt with in Section 2, giving rise to the coordinatization for an icosamonohedron in Section 3. Then, through continuous deformation from the regular case, the question of which triangles may be used to construct icosamonohedra (particularly convex ones) is examined. A measure of uniqueness is demonstrated in Theorem 4.2, alongside a subtle asymmetry concerning the interaction of combinatorics and geometry in determining how a triangle can be used to construct an icosamonohedron. Two explicit convex scalene icosamonohedra are constructed in Sections 5 and 6. The first has right triangular protoface, and although this scalene right icosamonohedron (Section 5) was discovered by Banchoff and Strauss [BS], their conjecture of its uniqueness is proven here for the first time. The example presented in Section 6 is entirely new.

\section{Tricolorings}

In order to be able to construct any scalene icosamonohedron, it must be possible to color the edges of a regular icosahedron in such a way that each face has its three edges colored differently; each color then represents the length of the edge to which it is assigned. Such a labeling is called a tricoloring.

Proposition 2.1. There exists a tricoloring of the icosahedron. Furthermore, all tricolorings of the icosahedron are combinatorially equivalent.

Proof. Using only the rule that each triangle is tricolored, it is easy to check that the pattern in Fig. 1(i) is forced at each vertex of the icosahedron. Note that triangle $(*)$ in Fig. 1(i) is oriented differently from the rest. Every vertex contains exactly one such

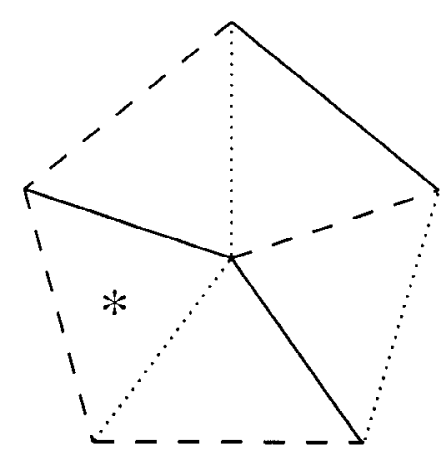

(i)

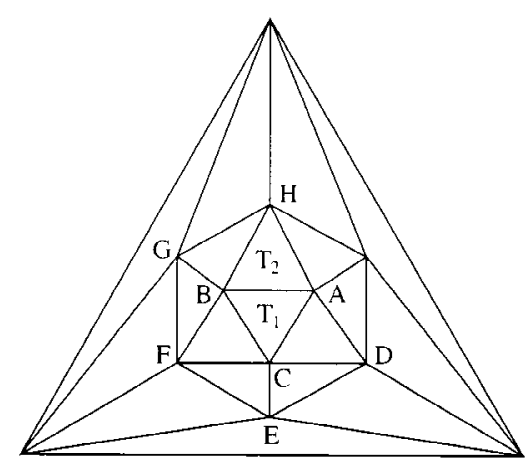

(ii)

Fig. 1. (i) Tricoloring at a vertex and (ii) Schlegel diagram of the icosahedron. 


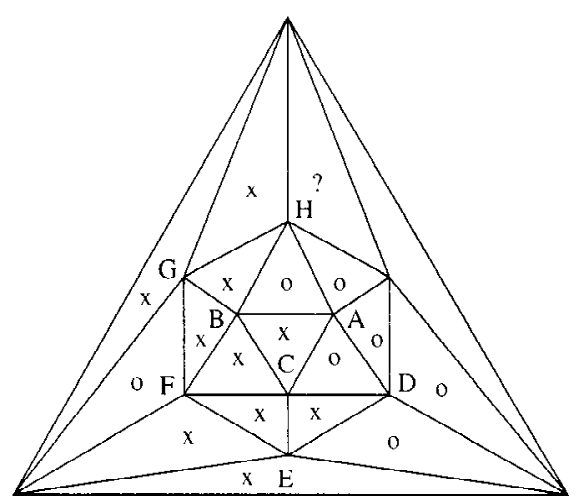

(i)

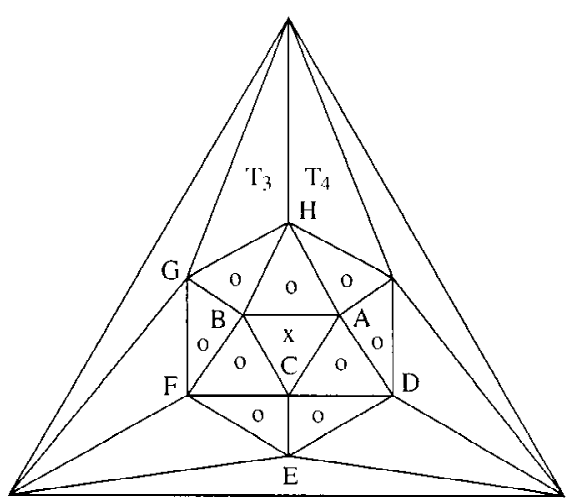

(ii)

Fig. 2. The two attempts to give orientations to the faces of an icosahedron.

triangle, and we call this triangle the minority triangle at that vertex. Furthermore, we may assume by symmetry that the central triangle $T_{1}$ in Fig. 1(ii) is the minority triangle of vertex $A$. Because $B$ contains $T_{1}$ and a triangle $T_{2}$ of opposite orientation, either $T_{1}$ or $T_{2}$ is the minority triangle of $B$.

If $T_{2}$ is the minority triangle at $B$, we examine (in order) the orientations of all triangles contained by vertices $B, C, D, E, F$, and $G$ which are forced by the rule that each vertex contains exactly one minority triangle. Then vertex $H$ is forced to have two triangles of one orientation and two of another, as in Fig. 2(i). If $T_{1}$ is the minority triangle at $B$, we find by the same process that either $T_{3}$ or $T_{4}$ of Fig. 2(ii) is the minority triangle at $H$. Neither choice leads to a contradiction; in fact, a choice of tricoloring for $T_{1}$ and minority triangle at $H$ determines uniquely a tricoloring of the entire Schlegel diagram. In particular, there are at most two tricolorings, namely, the two shown in Fig. 3. However, after reflecting and switching the dashed segments for the dotted ones, we see that these two diagrams are combinatorially equivalent, proving the proposition.

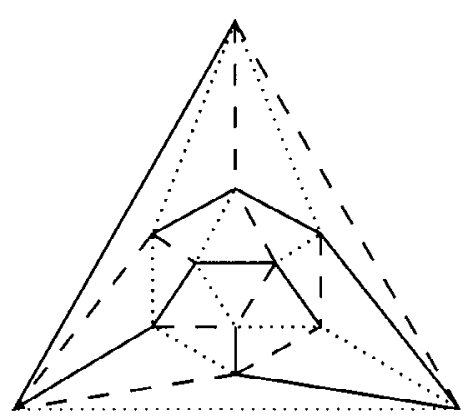

(i)

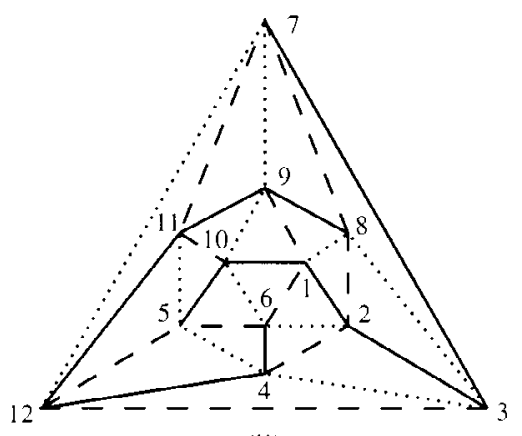

(ii)

Fig. 3. Two candidates for tricolorings of the icosahedron. 

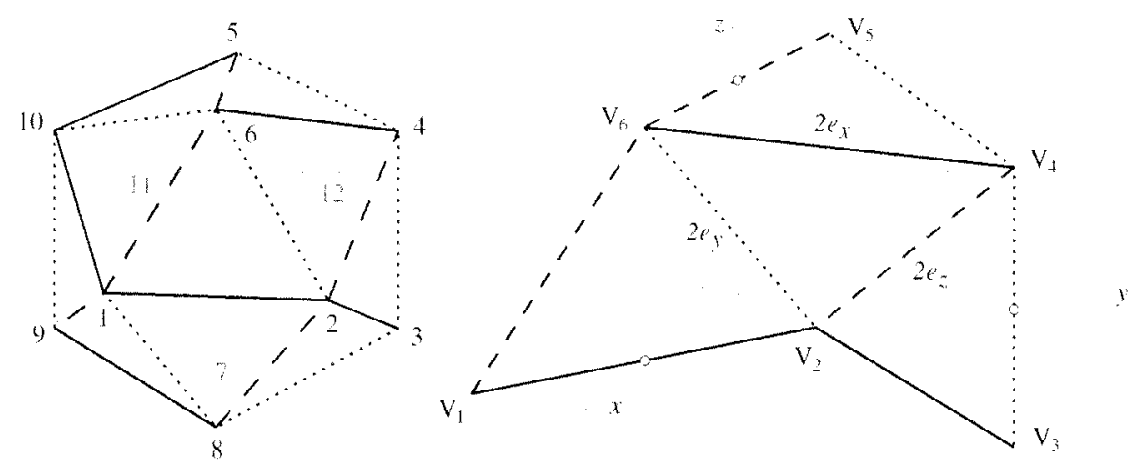

Fig. 4. Tricoloring and parametrization of the icosamonohedron.

\section{Coordinatization of Icosamonohedra}

The regular icosahedron may be thought of in coordinates as the convex hull of the 12 points

$$
\begin{array}{llll}
P_{1}(\tau,-1,0), & P_{2}(\tau, 1,0), & P_{11}(-\tau,-1,0), & P_{12}(-\tau, 1,0), \\
P_{3}(0, \tau,-1), & P_{4}(0, \tau, 1), & P_{9}(0,-\tau,-1), & P_{10}(0,-\tau, 1), \\
P_{5}(-1,0, \tau), & P_{6}(1,0, \tau), & P_{7}(-1,0,-\tau), & P_{8}(1,0,-\tau),
\end{array}
$$

where $\tau=(1+\sqrt{5}) / 2$. Edges such as $\overline{P_{1} P_{2}}$ which intersect coordinate axes are called axial segments, and may be referred to by the axis that they cross, so that $\overline{P_{1} P_{2}}$ is an $x$-axial segment. Due to symmetry of the regular icosahedron, we may take $D, E$, and $C$ from Fig. 2(ii) to be $P_{2}, P_{4}$, and $P_{6}$ respectively (superimposing Figs. 2(ii) and 3(ii)), thus fixing a tricoloring and coordinatization as in Figs. 3(ii) and 4. Half-turns about any of the coordinate axes fix this tricoloring (this is much easier to see on a three-dimensional representation, but Fig. 4 helps).

Any Euclidean motion $\varphi$ of $\mathbf{R}^{3}$ which induces a symmetry of a tricolored regular icosahedron also induces a symmetry of any convex icosamonohedron $P$ with that tricoloring, by the rigidity theorem of Cauchy [L, p. 60]: two 3-polytopes with congruent and similarly situated facets have equal dihedral angles. Indeed, the action of $\varphi$ on the tricolored regular icosahedron induces the desired correspondence between the facets of $P$ and those of $\varphi(P)$. In particular, half-turns about coordinate axes have this property. It follows that in any convex icosamonohedron, each axial segment remains parallel to the appropriate coordinate plane. In fact, if we let $V_{i}$ on an icosamonohedron correspond to $P_{i}$ on the regular figure, we may (by Proposition 2.1) coordinatize any convex scalene icosamonohedron (if it exists) by using only nine variables with nine constraints. The vertices are then:

$$
\begin{array}{llll}
V_{1}\left(x_{x},-y_{x},-z_{x}\right), & V_{2}\left(x_{x}, y_{x}, z_{x}\right), & V_{11}\left(-x_{x},-y_{x}, z_{x}\right), & V_{12}\left(-x_{x}, y_{x},-z_{x}\right), \\
V_{3}\left(-x_{y}, y_{y},-z_{y}\right), & V_{4}\left(x_{y}, y_{y}, z_{y}\right), & V_{9}\left(x_{y},-y_{y},-z_{y}\right), & V_{10}\left(-x_{y},-y_{y}, z_{y}\right), \\
V_{5}\left(-x_{z},-y_{z}, z_{z}\right), & V_{6}\left(x_{z}, y_{z}, z_{z}\right), & V_{7}\left(-x_{z}, y_{z},-z_{z}\right), & V_{8}\left(x_{z},-y_{z},-z_{z}\right) .
\end{array}
$$

In order for these to determine an icosamonohedron, we require all edges of the same color as the $\ell$-axial segment to have length $2 e_{\ell}$, where $\ell \in\{x, y, z\}$. Thus, with $d_{i, j}$ 
denoting the distance from $V_{i}$ to $V_{j}$, we have the equalities

$d_{1,2}=d_{2,3}=d_{4,6}=2 e_{x}, \quad d_{3,4}=d_{4,5}=d_{6,2}=2 e_{y}, \quad d_{5,6}=d_{6,1}=d_{2,4}=2 e_{z}$,

whence $\left(d_{2,3}\right)^{2}-\left(d_{2,4}\right)^{2}=4 e_{x}^{2}-4 e_{z}^{2} \Rightarrow$

$$
x_{x} x_{y}+z_{x} z_{y}=e_{x}^{2}-e_{z}^{2}
$$

$\left(d_{4,5}\right)^{2}-\left(d_{4,6}\right)^{2}=4 e_{y}^{2}-4 e_{x}^{2} \Rightarrow$

$$
x_{y} x_{z}+y_{y} y_{z}=e_{y}^{2}-e_{x}^{2}
$$

$\left(d_{6,1}\right)^{2}-\left(d_{6,2}\right)^{2}=4 e_{z}^{2}-4 e_{y}^{2} \Rightarrow$

$$
y_{x} y_{z}+z_{x} z_{z}=e_{z}^{2}-e_{y}^{2}
$$

$2\left(d_{2,3}\right)^{2}+2\left(d_{2,4}\right)^{2}-\left(d_{1,2}\right)^{2}-\left(d_{3,4}\right)^{2}=4 e_{x}^{2}-4 e_{y}^{2}+8 e_{z}^{2} \Rightarrow$

$$
x_{x}^{2}+y_{y}^{2}-2 y_{x} y_{y}=e_{x}^{2}-e_{y}^{2}+2 e_{z}^{2}
$$

$2\left(d_{4,6}\right)^{2}+2\left(d_{4,5}\right)^{2}-\left(d_{3,4}\right)^{2}-\left(d_{5,6}\right)^{2}=8 e_{x}^{2}+4 e_{y}^{2}-4 e_{z}^{2} \Rightarrow$

$$
y_{y}^{2}+z_{z}^{2}-2 z_{y} z_{z}=e_{y}^{2}-e_{z}^{2}+2 e_{x}^{2}
$$

$2\left(d_{6,2}\right)^{2}+2\left(d_{6,1}\right)^{2}-\left(d_{1,2}\right)^{2}-\left(d_{5,6}\right)^{2}=-4 e_{x}^{2}+8 e_{y}^{2}+4 e_{z}^{2} \Rightarrow$

$$
x_{x}^{2}+z_{z}^{2}-2 x_{x} x_{z}=e_{z}^{2}-e_{x}^{2}+2 e_{y}^{2}
$$

$\left(d_{1,2}\right)^{2}=4 e_{x}^{2} \Rightarrow$

$$
y_{x}^{2}+z_{x}^{2}=e_{x}^{2}
$$

$\left(d_{3,4}\right)^{2}=4 e_{y}^{2} \Rightarrow$

$$
x_{y}^{2}+z_{y}^{2}=e_{y}^{2}
$$

and $\left(d_{5,6}\right)^{2}=4 e_{z}^{2} \Rightarrow$

$$
x_{z}^{2}+y_{z}^{2}=e_{z}^{2} .
$$

In view of Fig. 4 , we may assume that $x_{x}, y_{y}$, and $z_{z}$ are $\geq 0$. Furthermore, it is clear from the tricolored Schlegel diagram Fig. 3(ii) that an icosamonohedron collapses to a line segment if one of the edges has length zero. We therefore assume henceforth that $e_{x} e_{y} e_{z} \neq 0$.

\section{Deformation}

Whether or not a triangle can be used to construct an icosamonohedron depends, by homothety of the icosamonohedron, only on the similarity class of the triangle. The set of similarity classes of triangles is the image of a closed region in the quotient of the 


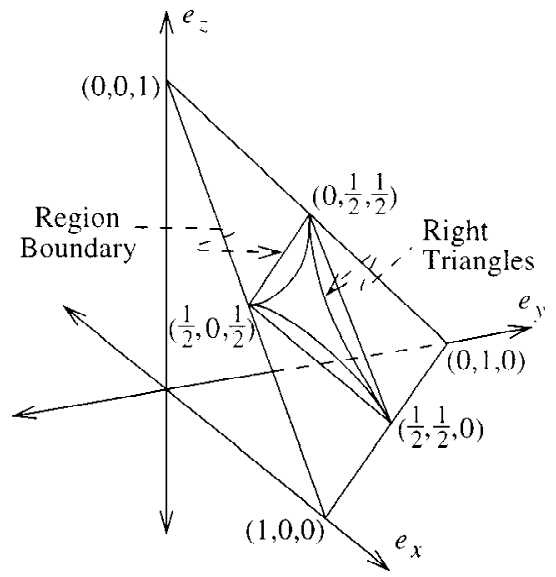

(i)

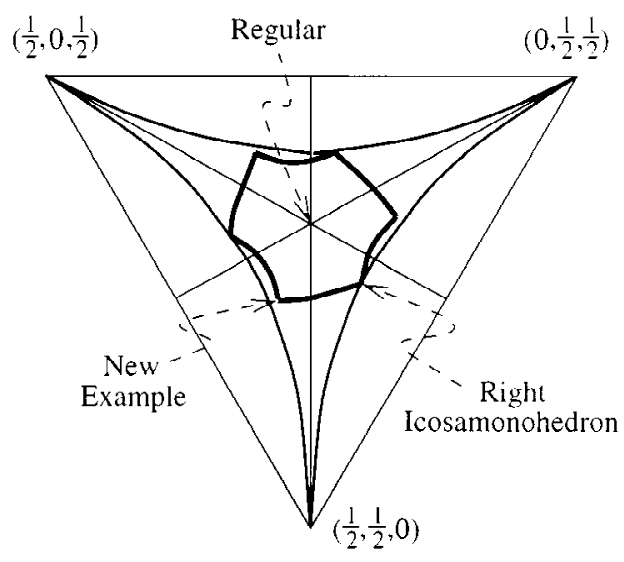

(ii)

Fig. 5. (i) The space of triangles. (ii) The space of triangles with the numerically determined border between triangles that give convex and nonconvex icosamonohedra.

real projective plane by an action of the symmetric group $S_{3}$ permuting a given set of coordinates. To view the set of triangles, we use an affine representation of this subset: for any ordered triple $(A, B, C)$ representing the edges of a triangle, we find a similar triangle $\left(e_{x}, e_{y}, e_{z}\right)=(1 /(A+B+C))(A, B, C)$ with $e_{x}+e_{y}+e_{z}=1$. Imposing the triangle inequalities on $e_{x}, e_{y}$, and $e_{z}$ yields a triangular set of points in the positive octant part of the plane $e_{x}+e_{y}+e_{z}=1$, depicted in Fig. 5. Each scalene triangle is represented in this region six times, by taking permutations of $e_{x}, e_{y}$, and $e_{z}$. The right triangles are defined by the cones $e_{x}^{2}+e_{y}^{2}=e_{z}^{2}, e_{y}^{2}+e_{z}^{2}=e_{x}^{2}$, and $e_{z}^{2}+e_{x}^{2}=e_{y}^{2}$, whose intersections with the plane $e_{x}+e_{y}+e_{z}=1$ are hyperbolas, also shown in Fig. 5.

The original motivation for the propositions proven in this paper stems from two numerical computer searches looking for convex solutions to (1)-(9) parametrizing icosamonohedra. The first search was conducted by Banchoff and Strauss in 1979 [BS], while the second search was completed by the author in 1993 using Mathematica [M]. Both searches were accomplished by starting at the equilateral triangle $\left(\frac{1}{3}, \frac{1}{3}, \frac{1}{3}\right)$ and moving outward along rays in the space of triangles, solving (1)-(9) numerically for icosamonohedral solutions until nonconvexity was detected.

When the original search was conducted, a faulty symmetry assumption was madenamely, that all orderings $\left(e_{x}, e_{y}, e_{z}\right)$ of the edges of a triangle induce congruent solutions to (1)-(9) via a rotation of the axes or a reflection. Consequently, only one-sixth of the space of triangles was searched (it happened to be a sixth that contained the right-triangle solution), and the other part was missed. After the author discovered the error, which is intimately related to the chirality of Lemma 4.1, the second search revealed that the apparent boundary of the set of triangles giving rise to convex solutions is a set of curves, illustrated in Fig. 5(ii). Note that the curves have symmetry of order three, not six.

Consequently, the two distinct types of boundary curves in Fig. 5(ii) intersect in two different kinds of points, each repeated three times. One kind of intersection point lies 
on a hyperbola representing right triangles, while the other kind does not. The object corresponding to the former is the unique icosamonohedron constructed from scalene right triangles, and is discussed in Section 5. Its existence was established by Banchoff and Strauss [BS], who described it synthetically (i.e., without coordinates-see the remark after Corollary 5.2). Its uniqueness, however, which is a manifestation of the subtle asymmetry (in fact, achirality) in the equations, is proven here for the first time. The other intersection point (see Section 6) was first found when the 1993 numerical search was conducted.

Before stating the main result of the paper (Theorem 4.2), we prove a lemma.

Lemma 4.1. A tricolored regular icosahedron is chiral (cannot be superimposed on its reflection), and has symmetry group $\mathbf{Z}_{2} \times \mathbf{Z}_{2}$, even if two of the colors are set equal.

Proof. Since half-turns about the coordinate axes in $\mathbf{R}^{3}$ fix any tricoloring by Section 3, it suffices to treat the case where two colors are set equal (stricly speaking, this is no longer a tricoloring). Chirality comes from the edges colored by the minority color, whose two connected components form a (piecewise linear) double helix, which is chiral. Furthermore, any symmetry of that double helix is a symmetry of the rectangle which is the convex hull of the minority-colored axial segments. Chirality then implies that no nontrivial symmetry can restrict to the identity on this rectangle, whence there can be at most four symmetries of this double helix.

In what follows, an icosamonohedron is called tricolored if its edge graph has a tricoloring with all edges of the same color having the same length. Although scalene icosamonohedra are automatically tricolored, it was indicated to the author by Branko Grünbaum that it is necessary to exclude isosceles cases such as Figure 28 of [G].

\section{Theorem 4.2. Let $T$ be a triangle.}

(i) At most two noncongruent tricolored convex icosamonohedra have protoface $T$. If $T$ is isosceles, then there is at most one. If $T$ is obtuse, then there are none.

(ii) For $T$ sufficiently close to regular, the number of tricolored convex icosamonohedra with protoface $T$ is precisely two if $T$ is scalene or one if $T$ is isosceles.

(iii) Unless $T$ is equilateral, the symmetry group of any tricolored convex icosamonohedron with protoface $T$ is isomorphic to the Klein 4-group $\mathbf{Z}_{2} \times \mathbf{Z}_{2}$.

Proof. Let $T$ have edges $\left\{e_{x}, e_{y}, e_{z}\right\}$. The result of Section 3 is that every convex tricolored icosamonohedron has a parametrization by (1)-(9). Therefore, $T$ can yield at most six such icosamonohedra: one for each permutation of $\{x, y, z\}$. It is easy to check, however, that cyclic permutations of $\{x, y, z\}$ induce cyclic permutations of (1)-(3), (4)-(6), and (7)-(9), and that any solutions thus obtained are congruent via a rotation with axis $(1,1,1)$ that cycles the coordinate axes. Thus there are at most two congruence classes of solutions for each triangle. Moreover, if two of $e_{x}, e_{y}$, and $e_{z}$ are equal, then transposition of the equal pair obviously induces the identity on (1)-(9). Finally, if $T$ has an obtuse angle which is (say) opposite the $x$-axial segment, then the angle sum at 
vertex $V_{6}$ is larger than $2 \pi$, so any icosamonohedron with protoface $T$ will fail to be convex locally at $V_{6}$. This proves (i).

The existence part of statement (ii) comes from an application of the Implicit Function Theorem [R, Theorem 9.28]. Equations (1)-(9) are all of the form $f_{i}(X)=g_{i}(Y)$ where $f_{i}: \mathbf{R}^{9} \rightarrow \mathbf{R}$ and $g_{i}: \mathbf{R}^{3} \rightarrow \mathbf{R}$. Define $h_{i}: \mathbf{R}^{12}=\mathbf{R}^{9} \times \mathbf{R}^{3} \rightarrow \mathbf{R}$ by $h_{i}(X, Y)=$ $f_{i}(X)-g_{i}(Y)$. Let

$$
\left(h_{1}, \ldots, h_{9}\right)=H: \mathbf{R}^{12} \rightarrow \mathbf{R}^{9} \quad \text { and } \quad\left(f_{1}, \ldots, f_{9}\right)=F: \mathbf{R}^{9} \rightarrow \mathbf{R}^{9} .
$$

The icosamonohedral solutions are the level set $\left\{W \in \mathbf{R}^{12} \mid H(W)=0\right\}$. Suppose that $H\left(X_{0}, Y_{0}\right)=0$ for some $X_{0} \in \mathbf{R}^{9}$ and $Y_{0} \in \mathbf{R}^{3}$, and that the derivative (i.e., Jacobian matrix) $F^{\prime}\left(X_{0}\right)$ is nonsingular. Applying the Implicit Function Theorem to $H$ implies that $X_{0}=G\left(Y_{0}\right)$, where $G$ is a differentiable function defined on some open set in $\mathbf{R}^{3}$ containing $Y_{0}$ and satisfying $H(G(Y), Y)=0$. Thus, if $Y_{0}$ gives the (ordered triple of) edge-lengths of a triangle, then $X_{0}$ gives the parametrization of the unique icosamonohedron built from triangles with (ordered) edges $Y_{0}$, and points close to $Y_{0}$ also determine unique parametrizations of icosamonohedra. It is now routine to check (by hand, even) that $F^{\prime}$ is nonsingular at the regular icosahedron.

For the last part of (ii), observe that the minority triangle (Section 2) at any vertex has the same orientation as the minority triangle at any other vertex, whence there are only four minority triangles all told. After switching two edge-colors, which changes the orientations of the minority triangles, any congruence with the original figure (colors not switched) must be orientation-reversing. Therefore, it is enough to show that switching two colors on a tricolored regular icosahedron and then reflecting yields two figures which cannot be superimposed in such a way that the colors match. Now, the endpoints of the two dotted components in Fig. 4 are the endpoints of the solid axial segments, which are the middle segments of the five-segment solid components. Switching solid and dashed segments does not preserve this combinatorial property.

Part (iii) is a consequence of Lemma 4.1 and the rigidity theorem of Cauchy.

\section{The Unique Scalene Right Icosamonohedron}

The purpose of this section is the answer (Theorem 5.1) to the question, "Which scalene right triangles can be used to build a convex icosamonohedron?" As a consequence, a refinement and extension of Theorem 4.2 are obtained in Corollary 5.2. Assume for the duration of this section that $\left(e_{x}, e_{y}, e_{z}\right)$ are the edges of a right triangle with $e_{x} e_{y} e_{z} \neq 0$. By cycling the axes if necessary, assume in addition that

$$
e_{x}^{2}=e_{y}^{2}+e_{z}^{2}
$$

Suppose that a convex scalene right icosamonohedron exists. Its foldout around $V_{6}$ will then be as in Fig. 6 , so that the angle sum at $V_{6}$ is exactly $2 \pi$. Thus $V_{6}$ must lie in the relative interior of a ridge, and there are only two ways that this is possible without inducing coplanarity of all 12 vertices or nonconvexity: let $\overline{V_{1} V_{5}}$ be a ridge (Case I), or let $\overline{V_{2} V_{10}}$ be a ridge (Case II), but not both. In Case I, $V_{1}$ and $V_{6}$ end up with the same $z$-coordinate, so $-z_{x}=z_{z}$; but $\overline{V_{2} V_{4}}$ remains parallel to $\overline{V_{6} V_{5}}$ so that also $z_{x}=z_{y}$. 

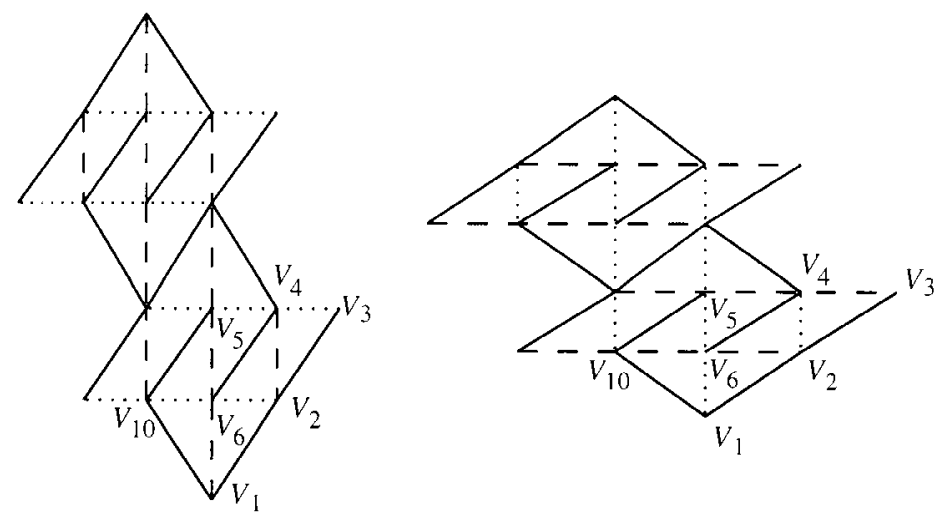

Fig. 6. Foldouts of a right icosamonohedron. Note that switching the dotted and dashed segments (i.e., edgelengths $e_{y}$ and $e_{z}$ ) leaves the angle sum at $V_{6}$ fixed. Those wishing to build a model of the right icosamonohedron should use the left foldout.

Therefore, in Case I, $-z_{x}=-z_{y}=z_{z}$. In Case II, on the other hand, $V_{2}, V_{4}, V_{5}$, and $V_{6}$ all lie in the same horizontal plane, so they have the same third coordinate; hence $z_{x}=z_{y}=z_{z}$ in Case II.

(The reader wishing to follow through the calculations in this section and the next would be well advised to examine Fig. 4 for geometrical content whenever it is found that some of the variables or their negatives must be zero or equal.)

Independent of which case is at hand, we have the following. Applying (10) and (1) gives $x_{x} x_{y}+z_{x} z_{y}=e_{y}^{2}$, so $z_{x}=z_{y}$ (which holds in both cases) yields

$$
\begin{aligned}
x_{x} x_{y}+z_{y}^{2} & =e_{y}^{2} \quad \text { since } \quad z_{x}=z_{y}, \\
x_{x} x_{y}+e_{y}^{2}-x_{y}^{2}=e_{y}^{2} & \text { by }(8), \\
x_{x} x_{y}-x_{y}^{2} & =0,
\end{aligned}
$$

and hence

$$
x_{x}=x_{y} \quad \text { or } \quad x_{y}=0 .
$$

Also, since $V_{2} V_{4} V_{5} V_{6}$ define a rectangle even after folding, we get

$$
\begin{aligned}
& x_{z}=\frac{1}{2}\left(x_{x}-x_{y}\right), \\
& y_{z}=\frac{1}{2}\left(y_{x}-y_{y}\right) .
\end{aligned}
$$

Furthermore, $V_{5}, V_{6}$, and $V_{1}$ always lie in a plane containing the $z$-axis, so that

$$
\left(x_{x},-y_{x}\right)=k\left(x_{z}, y_{z}\right)
$$

for some $k \in \mathbf{R}$. (Note that $\left(x_{z}, y_{z}\right) \neq(0,0)$ since $e_{z} \neq 0$.) If in (11) we assume $x_{x}=x_{y}$, we conclude from (12) that $x_{z}=0$, which implies $x_{x}=0$ by (14), and hence, by (11) again,

$$
x_{y}=0
$$


anyway. It follows by (8) that $x_{y}^{2}+z_{y}^{2}=z_{y}^{2}=e_{y}^{2}$ so $z_{x}^{2}=z_{y}^{2}=z_{z}^{2}=e_{y}^{2}$, and since $z_{z} \geq 0$, we also get

$$
z_{z}=e_{y} .
$$

From this, (7) yields $y_{x}^{2}+e_{y}^{2}=e_{x}^{2}$, and applying (10) gives

$$
y_{x}^{2}=e_{z}^{2}
$$

in all cases. We will need to divide by $y_{x}$ or $y_{z}$ later, so it is necessary to determine under what conditions they are not zero. If $y_{z}=0$, then (14) implies that $y_{x}=0$ as well, and hence $e_{z}=0$ by (17). However, we have assumed above that $e_{x} e_{y} e_{z} \neq 0$, so it follows that $y_{x} y_{z} \neq 0$. Lastly, since $x_{y}=0$ by (15), equations (2) and (10) imply

$$
y_{y} y_{z}=-e_{z}^{2} \text {. }
$$

\subsection{Case I: $-z_{x}=-z_{y}=z_{z}=e_{y}$}

Using (3) we get $y_{x} y_{z}-e_{y}^{2}=e_{z}^{2}-e_{y}^{2}$, so $y_{x} y_{z}=e_{z}^{2}$ (and hence $y_{z} \neq 0$ since $e_{z} \neq 0$ ); but then $y_{x} y_{z}=-y_{y} y_{z}$ by (18), and this implies that

$$
y_{y}=-y_{x} .
$$

Applying (13) now yields $y_{z}=y_{x}$. Since $y_{y} \geq 0$ by the comment after (9), we get

$$
-y_{x}=-y_{z}=y_{y}=e_{z} \text {. }
$$

Using this last result in (9) gives $x_{z}=0$ and hence $x_{x}=x_{y}=0$ by (15) and (12). The complete solution in Case $\mathrm{I}$ is therefore

$$
x_{x}=x_{y}=x_{z}=0, \quad-y_{x}=y_{y}=-y_{z}=e_{z}, \quad-z_{x}=-z_{y}=z_{z}=e_{y} .
$$

All 12 vertices lie in a plane, and a picture is easily drawn (it is a rectangle).

\subsection{Case II: $z_{x}=z_{y}=z_{z}=e_{y}$}

Note that the subsection title is justified by (16). Using (5) and (10) we get

$$
y_{y}^{2}=4 e_{y}^{2}+e_{z}^{2} .
$$

Equations (3) and (10) imply

$$
y_{x} y_{z}=e_{z}^{2}-2 e_{y}^{2} .
$$

Use the comments before (18) to divide the left and right sides of (18) by those of (20); then square, and substitute with (17) and (19) to obtain the relation

$$
\frac{4 e_{y}^{2}+e_{z}^{2}}{e_{z}^{2}}=\left(\frac{-e_{z}^{2}}{e_{z}^{2}-2 e_{y}^{2}}\right)^{2}
$$


Cross-multiplying gives $e_{z}^{6}=\left(4 e_{y}^{2}+e_{z}^{2}\right)\left(e_{z}^{4}-4 e_{y}^{2} e_{z}^{2}+4 e_{y}^{4}\right)$ and hence $0=4 e_{y}^{6}-3 e_{y}^{4} e_{z}^{2}$, yielding $e_{y}=0$ or $4 e_{y}^{2}=3 e_{z}^{2}$. Since we assumed that $e_{x} e_{y} e_{z} \neq 0$, we conclude that

$$
e_{y}=\frac{\sqrt{3}}{2} e_{z}
$$

Arbitrarily setting $e_{z}=1$ and solving for the remaining variables, we get

$$
\begin{aligned}
& \left(\left(x_{x}, y_{x}, z_{x}\right),\left(x_{y}, y_{y}, z_{y}\right),\left(x_{z}, y_{z}, z_{z}\right)\right) \\
& =\left(\left(\sqrt{3}, 1, \frac{\sqrt{3}}{2}\right),\left(0,2, \frac{\sqrt{3}}{2}\right),\left(\frac{\sqrt{3}}{2},-\frac{\sqrt{3}}{4}, \frac{\sqrt{3}}{2}\right)\right),
\end{aligned}
$$

and (10) implies that $\left(2 e_{x}, 2 e_{y}, 2 e_{z}\right)=(\sqrt{7}, \sqrt{3}, 2)$. This proves the main result of this section, which is a conjecture of Banchoff and Strauss [BS]:

Theorem 5.1. There is a unique nonplanar convex icosamonohedron with scalene right triangular protoface, and its edges have lengths $(\sqrt{7}, \sqrt{3}, 2)$, up to scaling.

\section{Corollary 5.2.}

(i) There exists a scalene triangle which is the protoface of precisely one convex icosamonohedron.

(ii) There exist nonconvex scalene icosamonohedra with obtuse, right, and acute faces.

Proof. The $(\sqrt{7}, \sqrt{3}, 2)$ triangle satisfies (i) by the uniqueness part of Theorem 5.1. For (ii), use the Implicit Function Theorem as in the proof of Theorem 4.2(ii), checking invertibility of the Jacobian matrix $F^{\prime}$ at (21) by computer. Any nearby right triangles will give icosamonohedral solutions to (1)-(9) which must be nonconvex by Theorem 5.1. Furthermore, $F^{\prime}$ will remain nonsingular at these nearby right triangles, so that there will be acute triangles near these giving nonconvex solutions. Finally, any obtuse triangle near $(\sqrt{7}, \sqrt{3}, 2)$ must give a nonconvex solution by nonsingularity of $F^{\prime}$ and Theorem 4.2(i).

\section{Remark 5.3.}

1. There is an isosceles right icosamonohedron appearing in the family of Fig. 28 of [G], so the uniqueness in Theorem 5.1 depends on the adjective "scalene" as well as "right."

2. The parallel projection of the right icosamonohedron from the direction $(0,0,1)$ is a regular hexagon. The triangle $(\sqrt{7}, \sqrt{3}, 2)$ shows up in the projection as half of the rectangular region obtained by intersecting two distinct parallel diagonals of the hexagon with the main diagonal perpendicular to them. 


\section{Another Explicit Example}

This section is different from Section 5 in that the assumptions we make are not about the edge-lengths of the triangle we are using; the assumptions are instead concerned with conditions on the nine variables implied by assuming coplanarity of certain pairs of faces. We then derive conditions on the edge-lengths which imply the existence and uniqueness of the object in question. Let $\left(e_{x}, e_{y}, e_{z}\right)$ be the edge-lengths of a triangle, where again $e_{x} e_{y} e_{z} \neq 0$. Dividing by $e_{x}$, we assume for the duration of this section that $e_{x}=1$.

Theorem 6.1. There is a unique nonplanar convex scalene icosamonohedron which has $(*)\left\{V_{1}, V_{2}, V_{4}, V_{6}\right\}$ coplanar and $(* *)\left\{V_{4}, V_{5}, V_{6}, V_{10}\right\}$ coplanar.

Proof. Assume the conditions of the theorem. The conditions (*) and (**) imply that $\left\{V_{1}, V_{2}, V_{4}, V_{6}\right\}$ and $\left\{V_{4}, V_{5}, V_{6}, V_{10}\right\}$ are the vertices of parallelograms. Thus $(* *) \Rightarrow$

$$
z_{y}=z_{z}
$$

whence the fact that $(*)$ implies $\overline{V_{1} V_{2}}$ and $\overline{V_{6} V_{4}}$ are parallel yields

$$
z_{x}=0
$$

This forces $\overline{V_{1} V_{2}}$, and hence also $\overline{V_{6} V_{4}}$, to be parallel to the $y$-axis, so

$$
x_{y}=x_{z}
$$

Now $y_{x}^{2}=e_{x}^{2}=1$ by (7), and we wish $y_{x}>0$. However, if $y_{x}<0$, then we can have none of the three possibilities $y_{z}<y_{y}, y_{z}=y_{y}$, and $y_{z}>y_{y}$. Indeed, the first forces the convex hull of $\left\{V_{1}, V_{2}, V_{4}, V_{6}\right\}$ to be a rectangle and the angle opposite an edge of length $2 e_{z}$ to be a right angle, which is impossible since $(*)$ is not satisfied by the unique nonplanar convex right icosamonohedron. The equality $y_{z}=y_{y}$ is ruled out because $e_{x} \neq 0$. Finally, $y_{z}>y_{y}$ forces $\overline{V_{2} V_{6}}$ to be the long diagonal of a parallelogram, whence the angle opposite it is obtuse, which is ruled out by Theorem 4.2(i). Therefore

$$
y_{x}=1 \text {. }
$$

The above equalities are used so often throughout this section that they are not referred to explicitly. By (3), we have

$$
y_{z}=e_{z}^{2}-e_{y}^{2} .
$$

Since $d_{1,2}=d_{4,6}=2$, we get

$$
y_{y}=y_{z}+2 .
$$

Equation (1) yields $x_{x} x_{y}=1-e_{z}^{2}$, and adding twice this result to (6) yields

$$
x_{x}^{2}+z_{y}^{2}=1+2 e_{y}^{2}-e_{z}^{2}
$$


from which adding (5) and subtracting (4) gives $2 y_{y}=2+4 e_{y}^{2}-4 e_{z}^{2}$, so $y_{y}=1+2 e_{y}^{2}-$ $2 e_{z}^{2}$, and thus

$$
y_{z}=-1+2 e_{y}^{2}-2 e_{z}^{2}
$$

by (23). Subtracting (22) from this last result gives $0=-1+3 e_{y}^{2}-3 e_{z}^{2}$, implying that

$$
e_{z}^{2}=e_{y}^{2}-\frac{1}{3} .
$$

Replacing this back into (22) and (23) shows that

$$
y_{z}=-\frac{1}{3} \quad \text { and } \quad y_{y}=\frac{5}{3} .
$$

Equation (2) now implies that

$$
x_{y}^{2}=-\frac{4}{9}+e_{y}^{2}
$$

while (4), (25), and (26) give

$$
x_{x}^{2}=\frac{8}{9}+e_{y}^{2} .
$$

Multiplying the previous two results gives the same left-hand side as squaring (1); upon eliminating $x_{x}^{2} x_{y}^{2}$ and using (25) to substitute for $e_{z}^{2}$, we have

$$
\begin{aligned}
& \left(e_{y}^{2}-\frac{4}{9}\right)\left(e_{y}^{2}+\frac{8}{9}\right)=\left(\frac{4}{3}-e_{y}^{2}\right)^{2} \\
& \Rightarrow \quad e_{y}^{4}+\frac{4}{9} e_{y}^{2}-\frac{32}{81}=e_{y}^{4}-\frac{8}{3} e_{y}^{2}+\frac{16}{9} \\
& \Rightarrow \quad e_{y}^{2}=\frac{44}{63} \\
& \Rightarrow \quad e_{z}^{2}=\frac{23}{63}
\end{aligned}
$$

by (25). It is a simple matter to solve for the remaining variables; doing so and multiplying the solution by $\sqrt{63}$ yields

$$
\begin{aligned}
& \left(\left(x_{x}, y_{x}, z_{x}\right),\left(x_{y}, y_{y}, z_{y}\right),\left(x_{z}, y_{z}, z_{z}\right)\right) \\
& \quad=((10,3 \sqrt{7}, 0),(4,5 \sqrt{7}, 2 \sqrt{7}),(4,-\sqrt{7}, 2 \sqrt{7}))
\end{aligned}
$$

as a parametrization of the unique convex icosamonohedron with the desired properties, thus proving the theorem.

Remark 6.2. This figure has $(3 \sqrt{7}, 2 \sqrt{11}, \sqrt{23})$ protoface, which looks somewhat like the right triangle of Section 5. The Jacobian matrix $F^{\prime}$ from the proofs of Theorem 4.2(ii) and Corollary 5.2 is also nonsingular here, guaranteeing that perturbations still give icosamonohedra; it is likely that the resulting deformations of the example in this section are nonconvex in certain directions and convex in others.

\section{Further Directions}

Just as the assumptions in Sections 5 and 6 allowed explicit solution of (1)-(9), it may also be possible to solve for the two kinds of limiting isosceles icosamonohedra. One could also ask for a more explicit description of the boundary curves in Fig. 5. Of course, all of this is a special case of the much more general 


\section{Problem 7.1.}

(i) Classify all combinatorial types of convex monohedra. Find all combinatorial types which have a realization with trivial facet subdivisions.

(ii) Decide, for each combinatorial type, which congruence classes of faces can be used to construct it.

(iii) Decide, for each convex polygon $T$, which convex monohedra have protoface $T$.

As is evidenced by the present work, this problem can be a fertile ground for an interplay between combinatorics and Real algebraic geometry-after all, the collection of convex monohedra is just a semialgebraic set.

The unsubdivided condition in (i) is there to prevent things like tessellating the faces of an already known monohedron, although doing so and then perturbing the faces may be a fruitful way to find more convex monohedra. On the other hand, there are combinatorial types for which convex monohedra exist but any realization has nontrivial subdivisions. For instance, a tricolored octahedron with tetrahedral caps on a pair of opposite facets can be realized as a convex monohedron, but any such realization is really a subdivided parallelepiped. The tricolored icosahedron, on the other hand, obviously has an unsubdivided realization (the regular one), and every sufficiently small deformation as in Theorem 4.2 is still unsubdivided.

Another direction for further research is provided by a theorem in Section 3 of Chapter IV in [A] which states that every foldout homeomorphic to the sphere and having angle sums $\leq 2 \pi$ at each vertex can be glued to form a polytope. This provides existence, but there is no guarantee as to what the ridges and facets will be. Thus, although any acute (or right) triangle makes two foldouts similar to those in Fig. 6, and these have (after identification) angle sums $\leq 2 \pi$ with finitely many points of angle sum $<2 \pi$, Corollary 5.2 nevertheless guarantees that the realized polytopes of many of these foldouts cannot have the expected ridges. Instead, the realized polytopes will, for the nonobtuse triangles $T$ of Corollary 5.2(ii), be convex spheres isometric to the nonconvex icosamonohedron with face $T$ and tessellated by (nonplanar and not necessarily congruent) surfaces isometric to $T$. This motivates the following:

Problem 7.2. Describe, for each triangle, the facets of the convex realizations guaranteed by the foldout theorem for the two icosamonohedral foldouts.

For instance, Banchoff and Strauss [BS] found a polytope whose boundary is isometric to an icosamonohedral foldout using $(\sqrt{11}, \sqrt{7}, 2)$ right triangles but which has only eight facets, some ridges of which fail to be unions of edges in the tricoloring.

\section{Acknowledgments}

I would like to express my gratitude to Professor Thomas F. Banchoff for introducing me to his joint results with Strauss on this subject in his Topics in Geometry course at Brown University in the Spring of 1993, and for his direction and help in writing a previous version of this paper, which served as my undergraduate honors thesis at Brown. I am 
also indebted to Branko Grünbaum, who pointed out an oversight in the statement of Theorem 4.2.

\section{References}

[A] A. D. Aleksandrov, Vypuklye Mnogogranniki, Gosudarstvennoye Izdatelstvo Tekhniko-teoreticheskoy Literatury, Moskva, 1950. [Convex Polyhedra, State Publishing House of Technical-theoretical Literature, Moscow, 1950.]

[BS] T. Banchoff and C. Strauss, Skew-Regular Simplicial Polyhedra-A Computer Graphics Approach, Notices of the American Mathematical Society, 26(6), issue 126 (October, 1979), Abstract 769-D2, p. A-559. (Abstract for an AMS regional meeting at Howard University; personal communication with Banchoff for content of presentation.)

[FW] H. Freudenthal and B. L. van der Waerden, Over een bewering van Euclides, Simon Stevin, 25 (19461947), 115-121.

[G] B. Grünbaum, Acoptic Polyhedra, Contemporary Mathematics, 223 (1999), 163-199.

[L] L. A. Lyusternik, Convex Figures and Polyhedra (trans. from Russian by T. Jefferson Smith), Dover, New York, 1963.

[M] Mathematica, computer software by Wolfram Research.

[R] W. Rudin, Principles of Mathematical Analysis, 3rd edn., McGraw-Hill, New York, 1976.

[Z] G. Ziegler, Lectures on Polytopes, Graduate Texts in Mathematics, Vol. 152, Springer-Verlag, New York, 1995.

Received January 27, 1999, and in revised form February 18, 1999. Online publication May 16, 2000. 